\title{
Mild Toxic Anterior Segment Syndrome Following Intravitreal Injection of Dexamethasone Implant
}

\author{
Ayse Gul Kocak Altintas ${ }^{1 *}$ and Mine Turkay ${ }^{2}$ \\ ${ }^{1}$ Associate Professor, Saglik Bilimleri University, Ulucanlar Eye Hospital, Ankara, \\ Turkey \\ ${ }^{2}$ Saglik Bilimleri University, Ulucanlar Eye Hospital, Ankara, Turkey \\ *Corresponding Author: Ayse Gul Kocak Altintas, Associate Professor, Saglik \\ Bilimleri University, Ulucanlar Eye Hospital, Ankara, Turkey.
}

Received: April 21, 2020

Published: May 18, 2020

(C) All rights are reserved by Ayse Gul Kocak

Altintas and Mine Turkay.

\section{Abstract}

Mild toxic anterior segment syndrome (TASS) developed after Sustained-release Dexamethasone Intravitreal Implant, during the early post-implantation period. Prompt treatment started with topical antibiotics and steroid eye drop after subconjunctival injection. The signs and symptoms of inflammation were resolved with total remission on post-implantation day 10.

To the best of our knowledge, the presented case is the first report with TASS following sustained-release dexamethasone intravitreal implant.

Keywords: Toxic Anterior Segment Syndrome; Dexamethasone Intravitreal Implant; Ozurdex; Inflammation

\section{Introduction}

Ozurdex (Allergan, Irvine, CA, USA) implant is an efficacious treatment alternative for macular edema secondary to diabetic retinopathy, central or branch retinal vein occlusion, and noninfectious uveitis. Ozurdex is a sustained-release biodegradable intravitreal preservative-free dexamethasone delivery system. It slowly releases a total of $0.7 \mathrm{mg}$ steroid into the vitreous cavity for up to 6 months [1-4].

Ozurdex implant has the risk of steroid-induced glaucoma, cataract development, and even migration of the dexamethasone implant into the anterior chamber. In addition, every intravitreal injection has several ocular complications associated with its use, such as subconjunctival hemorrhage, corneal epithelial erosion, injury to clear human lens and inflammation, infection, or endophthalmitis [3-6].

Toxic anterior segment syndrome (TASS) is characterized by acute noninfectious anterior segment inflammation that may occur after intraocular surgery. Although TASS most often occurs after cataract surgery, it has also been reported after any kind of intraocular intervention, including keratoplasty and posterior segment surgeries [7-11].

In this report, a patient who has a Toxic anterior segment reaction presented with acute inflammation in the anterior chamber
24 hours after dexamethasone intravitreal implant for treatment of diabetic macular edema. As far as we know, ours is the first report that developed Toxic anterior Segment Syndrome (TASS) after Ozurdex implantation.

\section{Case Report}

A 67-year-old man who had persistent bilateral CME secondary to diabetic retinopathy was treated with dexamethasone implant (DI) for five years. He received a total of 4 DI in each eye previously. Each injection was performed in the operating room with the rules of sterilization under the topical anesthesia. A periocular cleaning was performed using $10 \%$ povidone-iodine and after the insertion of a sterile lid speculum, $5 \%$ povidone-iodine was placed on the conjunctival sac for 5 minutes and then cleaned with irrigation before the injection. Ozurdex implant was applied $3.5 \mathrm{~mm}$ posterior to the limbus, through the pars plana with the pre-filled 22-gauge needle in his right eye. Following the subconjunctival injection moxifloxacin, prophylactic topical antibiotics were started. He had not demonstrated any problem with his previous treatment with Ozurdex implants in either eye.

One day after the procedure, on the regular first control, the patient presented with blurred vision without ocular pain or photophobia. His visual acuity (VA) dropped to hand movement in the right eye. On slit-lamp examination, significant anterior chamber reaction with +4 inflammatory white blood cells without hypopyon 
combined with keratic precipitates, moderate corneal edema, and vitritis was observed. Vitreous opacification was present on B-scan ultrasonography and optical coherence tomography (OCT).

A prompt treatment started with a combination of gentamicin and dexamethasone injection sub-conjunctivally, then moxifloxacin and prednisolone acetate eye drops were given every hour. Eight hours after the beginning of treatment, VA increased to $2 \mathrm{~m}$ finger counting. A minimal decrement of both anterior chamber reaction and corneal edema was observed in the next morning, and visual acuity increased to 0.1 on Snellen's chart. Topical antibiotic and steroid drops application were continued every two hours.

Two days after the DI, the signs and symptoms of inflammation were diminished, and the VA increased to 0.3 on Snellen chart. Topical medications were decreased; antibiotic was given five times a day while steroid was applied eight times a day. Five days later, anterior chamber reaction, keratic precipitates, and corneal edema were apparently decreased. On OCT evaluation, vitreous opacification and macular thickness (MT) were decreased, while VA increased to 0.4 . On day five, the topical antibiotic was ceased while steroids diminished and were given five times a day.

On post-intervention day 10 , the signs of inflammation resolved with total remission of corneal edema and keratic precipitates. The final VA was 0.4 in Snellen chart. A significant decrement in MT was seen in OCT.

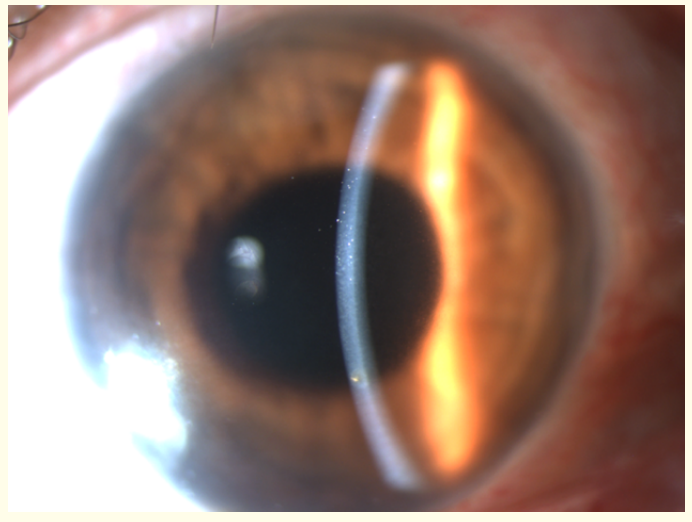

Figure 1a: Corneal edema and keratic presipitate before treatment of TASS.

\section{Discussion}

TASS, presented with severe sterile inflammation, is a very rare but one of the most feared complications of intraocular procedures,

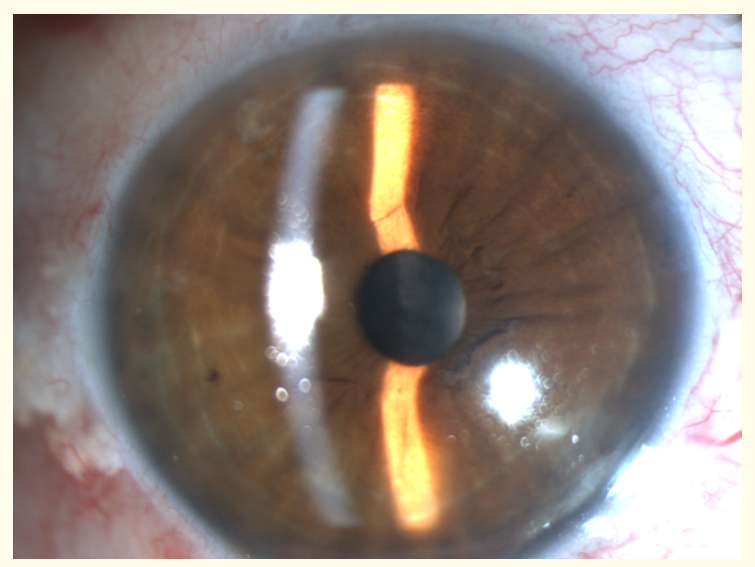

Figure 1b: Corneal edema and keratic prescipitates disappeared with the treatment.

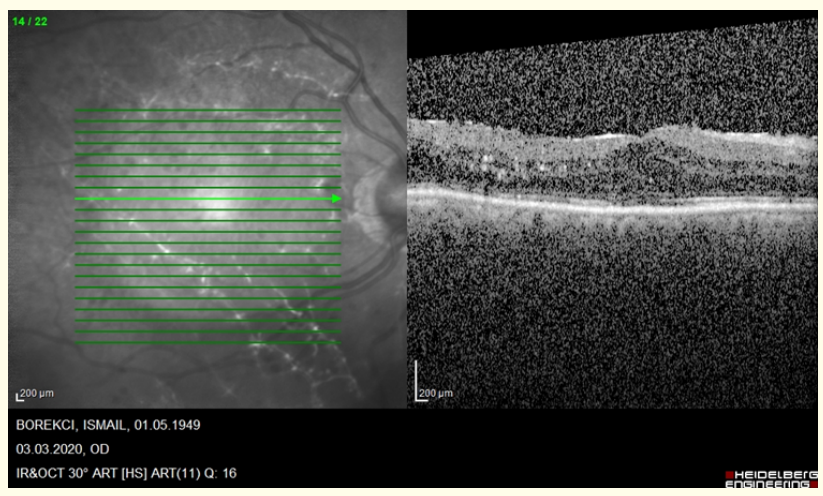

Figure 2a: Vitreous condensation in OCT at the diagnosis of TASS.

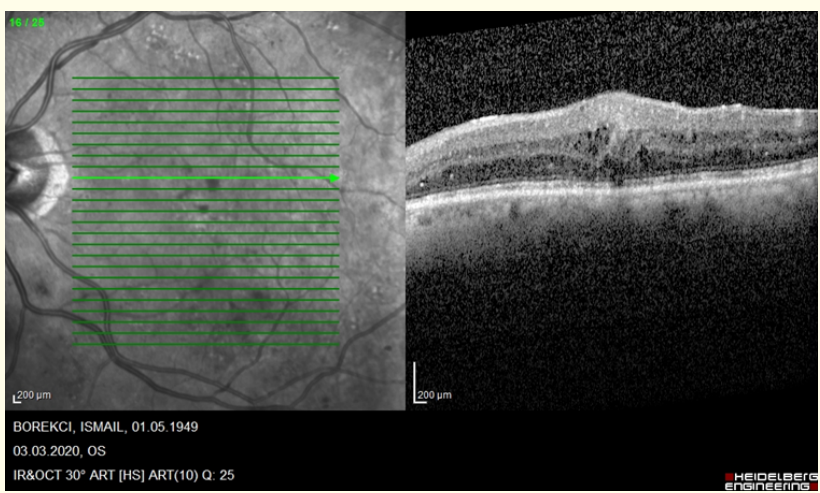

Figure 2b: Normal vitreous appearence in OCT comparing to fellow eye. 


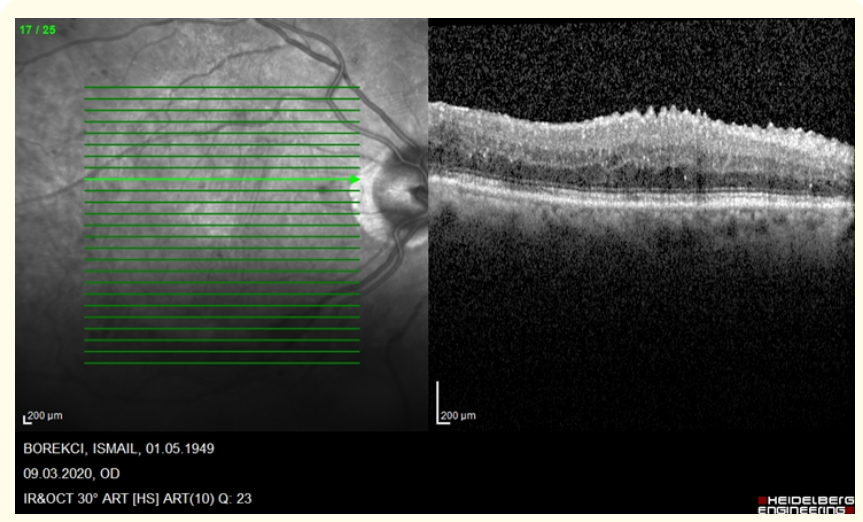

Figure 3a: Vitreous condancetion was disappered after the treatment of TASS.

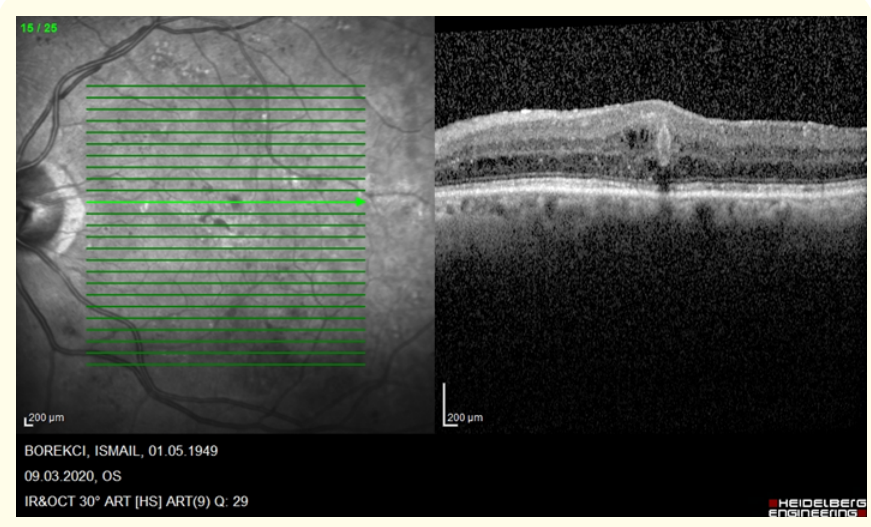

Figure 3b: Normal vitreous appearence to fellow eye.

including intravitreal injection. This noninfectious inflammation occurs from any kind of pharmacologic agents such as commonly used intracameral drugs including inadvertent dilution of adrenaline, phenylephrine, cefuroxime, lidocaine, abnormal pH or osmolality of intraocular preparations such as viscoelastic substance, any preservatives or disinfectant components such as povidoneiodine applied the conjunctival sac [12-17].

TASS generally resembles early postoperative bacterial endophthalmitis; therefore, differential diagnosis of TASS from infectious endophthalmitis is very important for correct treatment. Both conditions present with mild to severe corneal edema, keratic precipitates, anterior chamber reaction with or without hypopyon, and visual deterioration. In addition, infectious endophthalmitis present with ocular pain, conjunctival discharge, chemosis eyelid swelling. Sterile inflammation is severe and immediate nature, generally occurs within 12 - 48h of intraocular intervention. In contrast, infectious endophthalmitis occurs 4 - 7 days after the intraocular procedure [16-18].
Our case symptoms were noticed in regular post-injection control, 24 hours after the DI that the typical time of TASS starts. The patient presented with visual deterioration mild corneal edema, keratic precipitates, anterior chamber reaction without hypopyon. But fortunately, the symptoms and signs of infectious endophthalmitis such as ocular pain, conjunctival discharge, chemosis, eyelid edema were not observed.

Prompt control of inflammation with potent topical steroids, particularly $1 \%$, prednisolone acetate, or dexamethasone $0.1 \%$, is an essential treatment in TASS for the prevention of any irreversible ocular squeal to delicate ophthalmic structures. In some severe inflammation presented with hypopyon, oral prednisolone up to $40 \mathrm{mg}$ per day may be needed $[15,16]$. In contrast, fortified topical combined with or without intravitreal antibiotics is the main treatment method of bacterial endophthalmitis. Since TASS is not caused by sterility and contamination, it usually does not respond to antibiotics. However, most cases of unusual inflammation after the ophthalmic intervention should be regarded as infectious endophthalmitis until proven otherwise, and the use of broad-spectrum antibiotics such as moxifloxacin is recommended [15-19]. Even the symptoms of the presented patient resemble sterile inflammation; we prefer prompt initiation of topical antibiotics combined with a steroid.

In the presented case, anterior segment inflammation been controlled and mild corneal edema showed rapid clearing without any squeal, only with the intense ocular treatment without any systemic medication in a ten days period. Both anterior segment inflammation and corneal edema were not severe because the anterior chamber has not been entered, and implantation was performed posterior segment, so suspected toxic factor can only indirectly penetrate into the anterior segment.

The possible causative factor of inflammation in our cases may be insufficient irrigation and cleaning of povidone-iodine in the conjunctival sac. Since the Ozurdex implant has the content of 0.7 mg of preservative-free dexamethasone and made of the pre-filled delivery system, any contamination of drug and residue chemicals from preservatives were not possible.

Vitreous opacities were reported in less than a quarter of cases with TASS. Suzuki., et al [21]. observed vitreous opacity in $21.5 \%$ out of 241 cases with late-onset TASS, and it was reported in $23.8 \%$ in Oshika., et al. [22] series of 147 patient with subacute-onset of TASS. Both of these TASS series developed after the intraocular lens implantation. Michalska-Małecka., et al. [5] observed vitreous opacity in one case after intravitreal implantation of Ozurdex, out 
of 36 patients for macular edema treatment due to retinal vein occlusion. Anterior segment inflammation as a characteristic feature of TASS was not presented in their case.

In our case, according to successive OCT evaluation, vitreous inflammation presented with vitreous opacity occurred and disappeared in the same period with anterior segment inflammation.

\section{Conclusion}

As far as we know, presented case with TASS after sustainedrelease dexamethasone intravitreal implant is not reported before because the steroid itself is an essential medication in the treatment of TASS. In addition to prompt treatment and close monitoring of the patient, intravitreal release of dexamethasone as a potent steroid causes the rapid response to inflammation even in the unknown source of TASS in our case.

\section{Bibliography}

1. Boyer DS., et al. "Three-year, randomized, sham-controlled trial of dexamethasone intravitreal implant in patients with diabetic macular edema”. Ophthalmology 121.10 (2014): 1904-1914.

2. Mayer WJ., et al. "Comparison of intravitreal bevacizumab upload followed by a dexamethasone implant versus dexamethasone implant monotherapy for retinal vein occlusion with macular edema". Ophthalmologica 228.2 (2012): 110-116.

3. Madi HA., et al. "Corneal graft failure due to migration of Ozur$\mathrm{dex}^{\mathrm{TM}}$ implant into the anterior chamber". The American Journal of Ophthalmology Case Reports 8 (2017): 25-27.

4. Noma H., et al. "Aqueous flare and inflammatory factors in macular edema with central retinal vein occlusion: a case series". BMC Ophthalmology 11 (2013): 13-78.

5. Michalska-Małecka K., et al. "Evaluation of the effectiveness and safety of glucocorticoids intravitreal implant therapy in macular edema due to retinal vein occlusion". Clinical Interventions in Aging 11 (2016): 699-705.

6. Marchino T., et al. "Acute-onset endophthalmitis caused by alloiococcus otitidis following a dexamethasone intravitreal implant". Case Reports in Ophthalmology 4 (2013): 37-41.

7. Park CY., et al. "Toxic anterior segment syndrome-an updated review”. BMC Ophthalmology 18 (2018): 276.
8. Bodnar Z., et al. "Toxic anterior segment syndrome: update on the most common causes". Journal of Cataract and Refractive Surgery 38.11 (2012): 1902-1910.

9. Sevimli N., et al. "Toxic anterior segment syndrome following deep anterior lamellar keratoplasty". The Arquivos Brasileiros de Oftalmologia 79.5 (2016): 330-332.

10. Moisseiev E and Barak A. "Toxic anterior segment syndrome outbreak after vitrectomy and silicone oil injection". The European Journal of Ophthalmology 22.5 (2012): 803-807.

11. Andonegui J., et al. "Outbreak of toxic anterior segment syndrome after vitreous surgery". Archivos de la Sociedad Espanola de Oftalmologia 84.8 (2009): 403-405.

12. Sorenson AL., et al. "Toxic anterior segment syndrome caused by autoclave reservoir wall biofilms and their residual toxins". Journal of Cataract and Refractive Surgery 42.11 (2016): 16021614.

13. Bielory BP., et al. "Toxic anterior segment syndrome: inadvertent Administration of Intracameral Lidocaine 1\% and phenylephrine $2.5 \%$ preserved with $10 \%$ Benzalkonium chloride during cataract surgery". Cornea 36 (2017): 621-624.

14. Cakir B., et al. "Toxic anterior segment syndrome after uncomplicated cataract surgery possibly associated with intracameral use of cefuroxime". Clinical Ophthalmology 9 (2015): 493-497.

15. Litwin AS and Pimenides D. "Toxic anterior segment syndrome after cataract surgery secondary to subconjunctival gentamicin". Journal of Cataract and Refractive Surgery 38.12 (2012): 2196-2197.

16. Altintas AK., et al. "Toxic anterior segment syndrome outbreak after cataract surgery triggered by viscoelastic substance". Middle East African Journal of Ophthalmology 24.1 (2017): 4347.

17. Matsou A., et al. "Generic trypan blue as possible cause of a cluster of toxic anterior segment syndrome cases after uneventful cataract surgery". Journal of Cataract and Refractive Surgery 43.6 (2017): 848-852.

18. Lee SN. "Mild toxic anterior segment syndrome mimicking delayed onset toxic anterior segment syndrome after cataract surgery”. Indian Journal of Ophthalmology 62.8 (2014): 890892. 
19. Sorkin N., et al. "Evaluation of the retinal, choroidal, and nerve fiber layer thickness changes in patients with toxic anterior segment syndrome Graefes". Archive for Clinical and Experimental Ophthalmology 253.3 (2015): 467-475.

20. Marchino T., et al. "Acute-onset endophthalmitis caused by alloiococcus otitidis following a dexamethasone intravitreal implant". Case Reports in Ophthalmology 4 (2013): 37-41.

21. Suzuki T., et al. "Outbreak of late-onset toxic anterior segment syndrome after implantation of one-piece intraocular lenses". American Journal of Ophthalmology 159.5 (2015): 934-939.

22. Oshika T., et al. "Outbreak of subacute-onset toxic anterior segment syndrome associated with single-piece acrylic intraocular lenses". Ophthalmology 124 (2017): 519-523.

\section{Assets from publication with us}

- Prompt Acknowledgement after receiving the article

- Thorough Double blinded peer review

- Rapid Publication

- Issue of Publication Certificate

- High visibility of your Published work

Website: www.actascientific.com/

Submit Article: www.actascientific.com/submission.php Email us: editor@actascientific.com

Contact us: +919182824667 